\title{
Microfluidic Approaches for the Study of Emulsions: Transport of Solutes
}

Philipp Gruner ${ }^{1}$, Yousr Skhiri' ${ }^{2,3}$, Benoit Semin ${ }^{1 *}$, Quentin Brosseau ${ }^{1}$, Andrew D. Griffiths ${ }^{3}$, Valérie Taly ${ }^{2}$ and Jean-Christophe Baret ${ }^{1}$

${ }^{1}$ Max Planck Institute for Dynamics and Self-organization, Am Fassberg 17, Goettingen, Germany.

${ }^{2}$ Université Paris Descartes, 45 Rue des Saints-Pères, Paris, France.

${ }^{3}$ ISIS-CNRS-Université de Strasbourg, 8 allée Gaspard Monge, Strasbourg, France. *

Present address: ENS Paris, 45 Rue d'UIm, France.

This document is the last submitted version of the manuscript published as:

P. Gruner et al. Microfluidic Approaches for the Study of Emulsions: Transport of Solutes

MRS Online Proceedings Library (OPL) 1530, 2013

https://doi.org/10.1557/opl.2013.80

\section{ABSTRACT}

Molecular transport as an ageing process in emulsions is revisited using microfluidic droplet production, manipulation and analysis. We show how microfluidic systems provide extremely quantitative insights into the phenomenon. We designed microfluidic systems to address the specificity of molecular transport in fluorinated oils and showed the role of the surfactant solubilised in the oil phase on the time scale of the exchange and rationalize the effect of water soluble additives on the exchange rate. Finally, we also demonstrate that the droplet packing influences the exchange rate through the number of first neighbours. 


\section{INTRODUCTION}

Droplet-based microfluidic systems are promising platforms for high-throughput screening applications [1,2]. The versatile encapsulation of various types of biomaterials, such as proteins, nucleic acids, single genes, or cells makes droplets perfectly suitable as a tool for miniaturized and automatized enzymatic assays, cell screening, cancer diagnostics, or drug screening platforms [3-5]. The success of the technology relies on the very high-throughput of each individual step of droplet manipulation, which reaches several $\mathrm{kHz}$ for droplet production, reinjection, splitting, or fusion [6]. The droplets are used as microcontainers behaving in principle as independent, miniaturized and closed reacting vessels that can be actuated on demand for the steps required in a typical biological assay [1].

In practice, droplets are produced in microchannels and stabilised against coalescence by surfactant molecules, resulting in an emulsion, i.e. a dispersion of one phase into another, stabilised by surfactant molecules [7]. From a fundamental perspective, emulsions are systems out of equilibrium as they only correspond to a local minimum of the free energy landscape and the final equilibrium state where the two phases are separated in two compartments of macroscopic size will eventually be reached. The typical time-scale for this process is linked to the ageing mechanism of the emulsion, involving floculation, coalescence and molecular transport between droplets [7].

The first relevant step for the use of droplet as microreactor is the stabilisation of the droplets by the surfactant, which is achieved by formulation and optimisation of surfactant concentration and additives. This side is well established and microfluidic systems have proven very efficient tools to study the underlying mechanisms in the stabilisation of emulsions by surfactants $[8,9]$.

In the following, we will focus on the description of a second ageing mechanism which is crucial to be controlled, for most of the bio-assays, namely, the exchange of chemicals between droplets. Here the practical background is linked to storage and screening of chemicals and 
potential drugs in droplet-based microfluidics. This approach has already been demonstrated for various systems [4, 5], for example for screening of new drugs. Here, from a fundamental perspective, gradients in chemical potential between droplets will eventually level out, even if the absence of coalescence maintains the integrity of the reactors. The time scale of this process must be determined, in this somehow unusual emulsion system, where virtually all droplets can contain materials varying from droplet to droplet by composition or concentration (Figure 1).

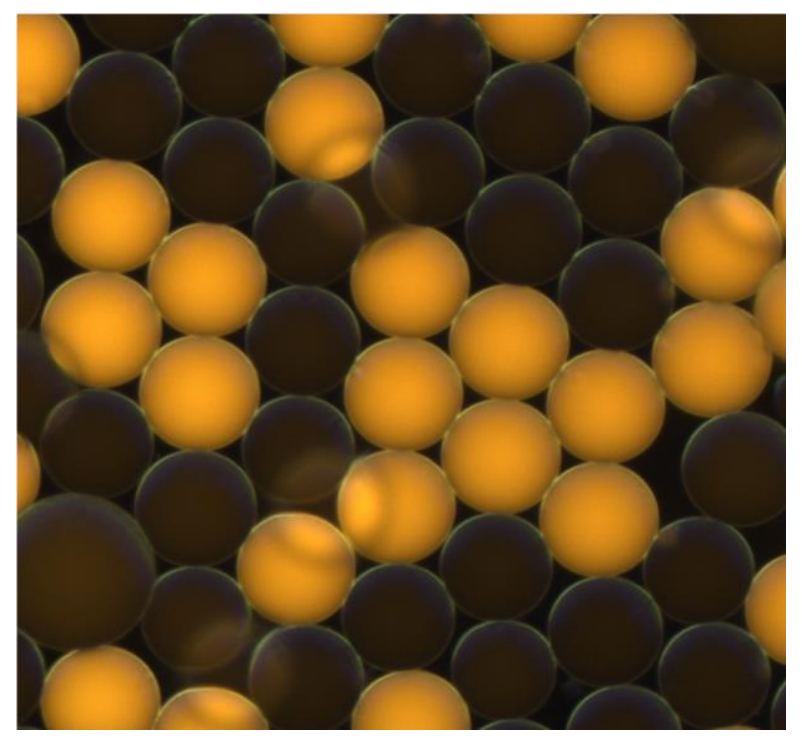

Figure 1: Droplet-based microfluidics provides tools to produce calibrated emulsion. They are used to determine the exchange rate of compounds. Example of an emulsion of 100 microns droplets containing sulforhodamine (bright droplets) or buffer (dark droplets). 


\section{EXPERIMENTS}

We designed microfluidic systems to address the specificity of molecular transport in fluorinated oils and showed the role of the surfactant solubilised in the oil phase on the time scale of the exchange and rationalize the effect of water soluble additives on the exchange rate. Finally, we also demonstrate that the droplet packing influences the exchange rate through the number of first neighbours [11].

\section{Microfluidic method}

We use standard lithography techniques to produce microfluidic chips for the formation of mixed emulsions made of monodisperse droplets of aqueous phases in fluorinated oils [12, 13]. Droplets of high dye concentration (typically $10 \mu \mathrm{M}$ ) are produced in one flow focussing junction and droplets without dye are produced in a second junction. The droplets are stabilized by block copolymer surfactants (PEG-PFPE, home made according to standard synthesis schemes [14]) to prevent coalescence. We interface the outlet of the chip with an incubation chamber, externally controllable for the loading of the emulsion, the storage from minutes to several hours and the droplet reinjection (Figure 2). As a model system, we use fluorescent compounds. The concentration of the dyes in the droplet directly relates to their concentrations. Using a laser induced fluorescent setup, we measure the fluorescence of every droplet of the emulsion for quantitative analysis of concentration over millions of droplets. Kinetic measurements are achieved by controlling the reinjection speed over several hours. 


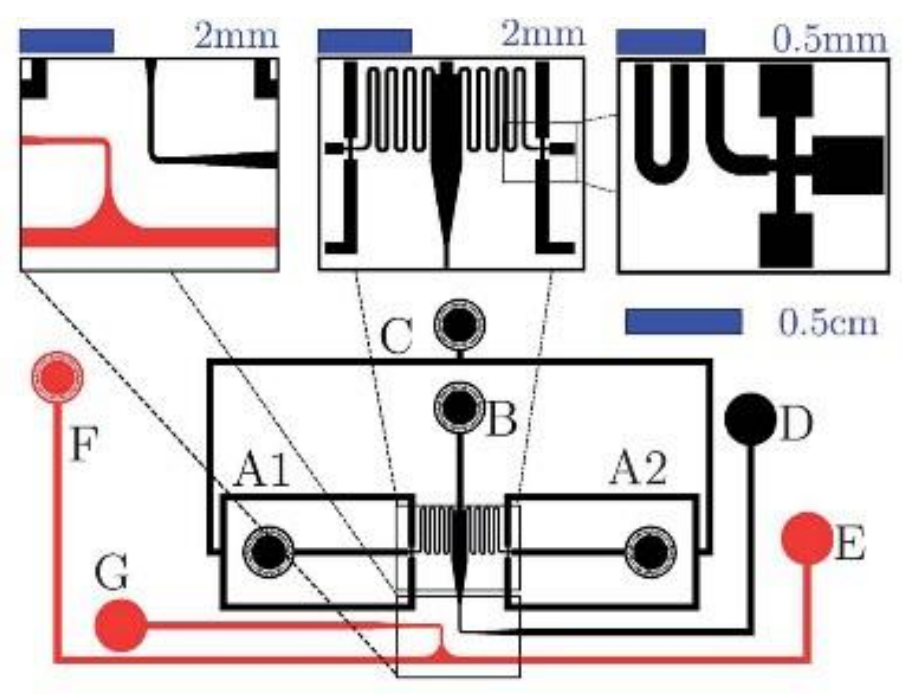

(a)

(b)

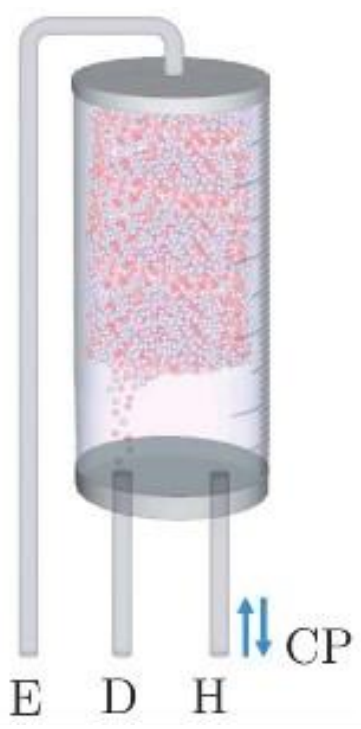

Figure 2: Sketch of the microfluidic chip for emulsification of mixed emulsion. Droplets containing a fluorescent dye (resorufin) are produced at one nozzle and droplet without the dye at the second nozzle. The droplets are stored in a reservoir for incubation and slowly reinjected for on-chip fluorescence measurement. Millions of droplets are measured over several hours for kinetic measurement of the exchange process. Reprinted with permission of the Royal Society of Chemistry (original publication: Y. Skhiri, P. Gruner et al. Soft Matter (2012) [11])

\section{RESULTS AND DISCUSSIONS}

\section{Effect of the surfactant on transport}

In a first set of experiments, we used resorufin in PBS buffer and monitor the exchange kinetics. The distribution of the droplet fluorescence at short time-scale (a few minutes) reveals to peaks, corresponding to the two types of droplets and as time increases the two concentrations tend to equilibrate.

A quantitative measurement of the exchange kinetics is performed by measuring the difference of concentration of the two populations as a function of time (Figure 3). The kinetic is affected by the concentration of the surfactant present in the oil phase: an increase of surfactant concentration leads to a decrease of the equilibration time-scale (increase of rate). The time scale 
of exchange is obtained by rescaling the time of all experiments by a single time scale, which turns out to be a linear function of surfactant concentration. The shape of the relaxation itself is a universal master curve showing an initial exponential relaxation asymptotically reaching a power-law in the late kinetic.

(a)

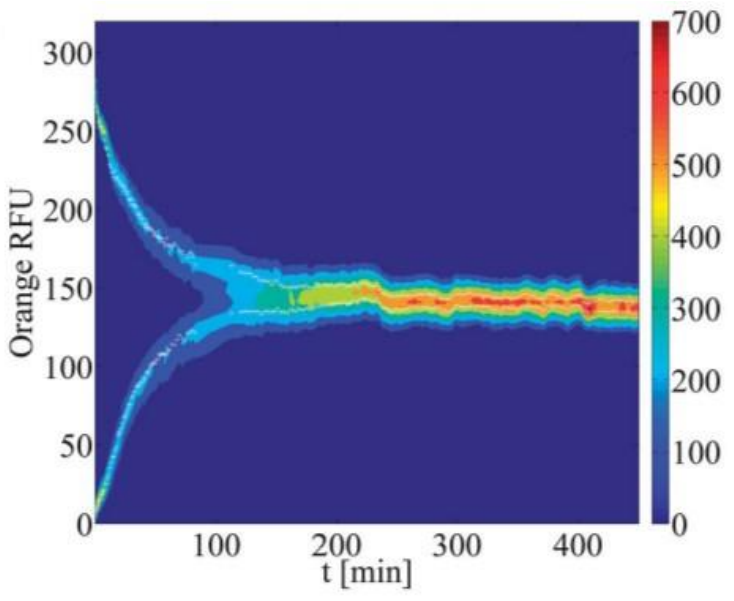

(c)

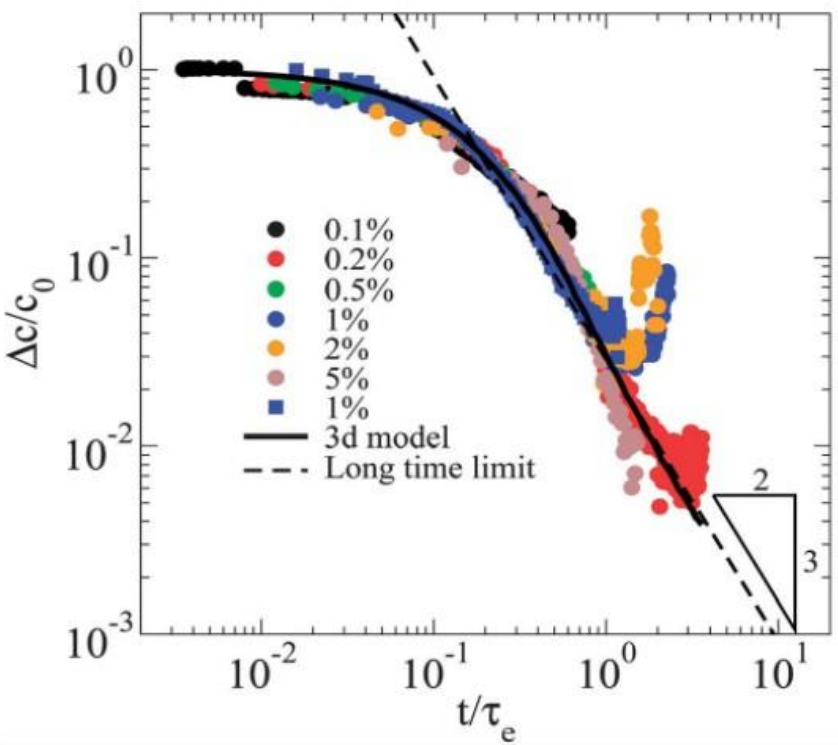

(b)

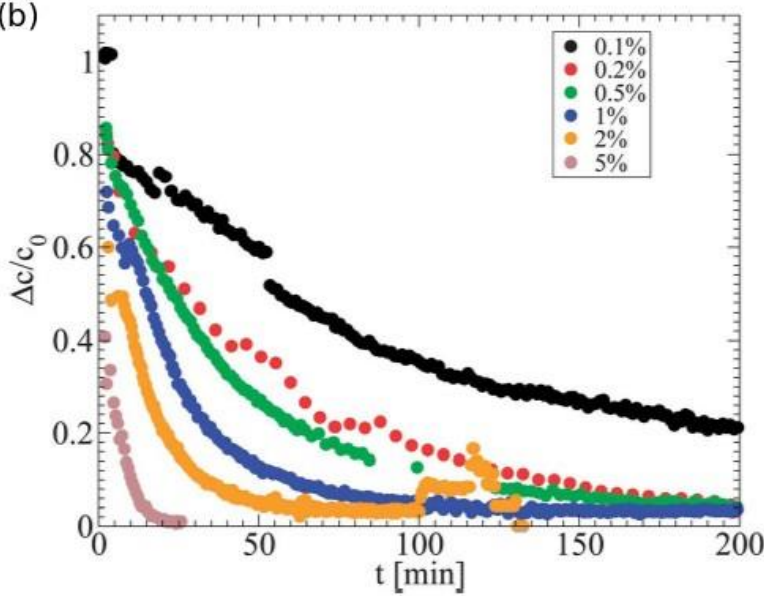

(d)

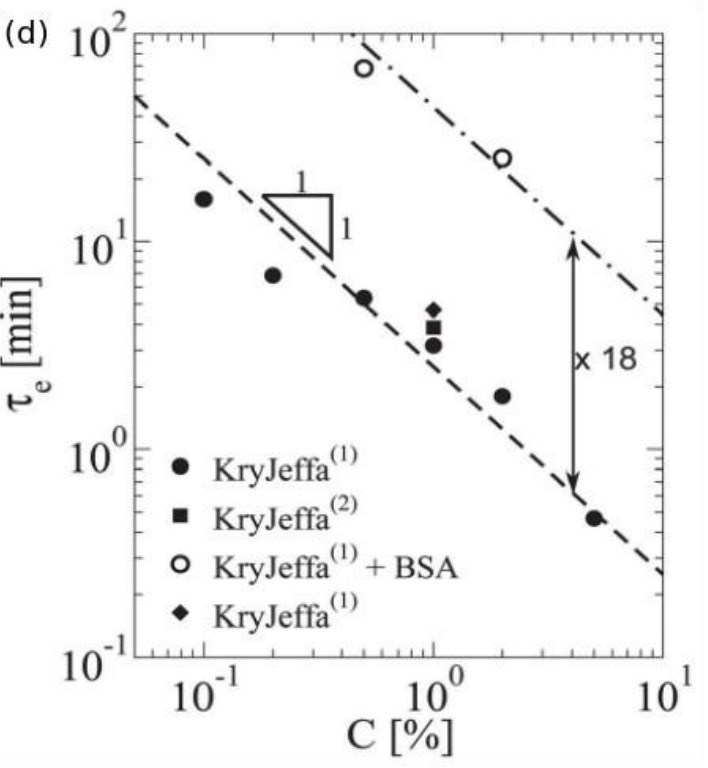

Figure 3: (a) Distribution of fluorescence in the emulsion as a function of time. Asymptotically, the droplet all contain the same concentration of dye. (b) Variation of the concentration in each population of droplets as a function of time and surfactant concentration: surfactant favors the exchange process. (c) Rescaling of the concentration difference on a master curve. The power law at late time is the result of the random distribution of the droplets in the emulsion. (d) Evolution 
of the exchange time scale as a function of surfactant concentration. Additives (BSA) are shown to decrease the exchange rate (empty dots). Reprinted with permission of the Royal Society of Chemistry (original publication: Y. Skhiri, P. Gruner et al. Soft Matter (2012) [11])

\section{Effect of chemistry and the role of additives}

The solubility of the dyes in the acqueous phase are known to influence the exchange [15]. As an example, we observed exchange rates of the order of one hour for resorufin, several days for fluorescein and a few seconds for coumarine. It is also known that additives such as the protein BSA (Bovine Serum Albumine) solubilised in the water droplet slows down the exchange kinetics [16] which we also observed (Figure 3d). However, the underlying mechanisms are not clear. One of the proposed mechanism is the creation of a layer of proteins at the water - oil interface that would create a kinetic barrier slowing down the exchange. In order to test this hypothesis, we have produced a mixed emulsion in which all droplets contain the same resorufin concentration. As the chemical potential of resorufin is in principle the same in all droplets, the concentration of resorufin should remain constant.

(a)

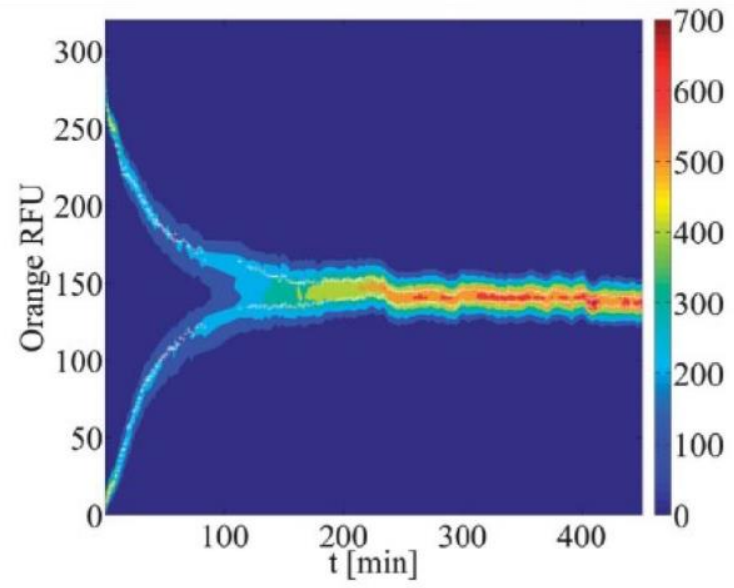

(b)

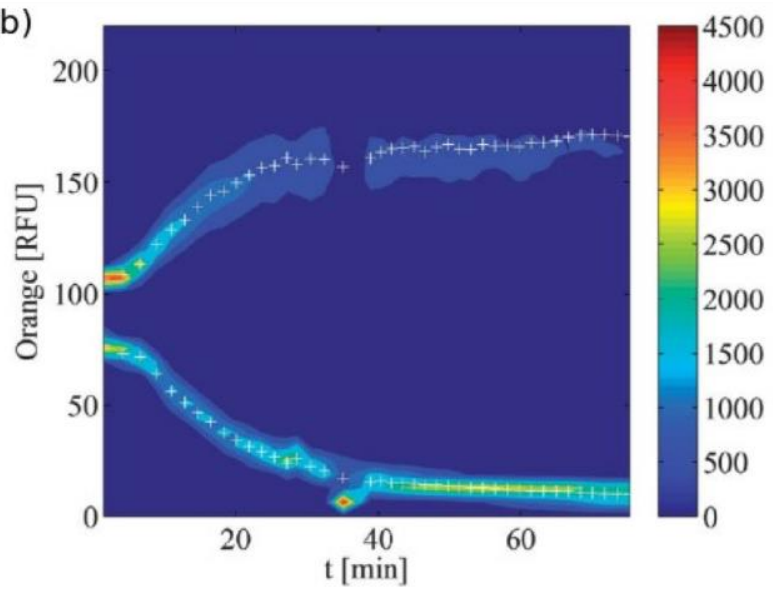

Figure 4: Comparison of the exchange process for an emulsion initially containing two different concentration of resorufin showing that concentrations equilibrate (a) and for an emulsion initially containing the same resorufin concentration but different concentration of the additive 
BSA showing that resorufin concentrates in BSA containing droplets (b). Reprinted with permission of the Royal Society of Chemistry (original publication: Y. Skhiri, P. Gruner et al. Soft Matter (2012) [11])

Here, we introduced BSA in half of the droplets and incubated and reinjected the emulsion. At short time scales, the fluorescence signal is the same in all droplets but the signal splits in two indicating that the BSA containing droplets and the non-BSA containing droplet contain different concentration of resorufin. There is therefore an exchange of dye and this exchange should not occur if BSA was only providing a kinetic barrier to exchange. BSA modifies the chemical potential of resorufin in the droplets (and therefore partition coefficient between the oil- surfactant mixture and the water) which impacts the kinetics of exchange in a process controlled by thermodynamic properties. Such an effect relates to the ability of BSA to bind a wide range of small molecules, such as potential drugs [17].

\section{Effect of packing and number of first neighbors during incubation}

The shape of the master curve also provides additional information on exchange mechanisms. Indeed, in a simple permeation model, two compartments would equilibrate with an exponential decay of the concentration difference [18]. The time scale is then given by partition coefficient, diffusion constant in the permeable layer and the spacing between the compartments. Our experiments display a more complex behaviour. In order to understand the shape of the master curve, one consider a regular array of droplets all separated by a permeable

membrane (Figure 5). Under these conditions, the concentration is given by a discrete diffusion equation. The eigenmode and eigenvalues of the Laplace equation can be analytically derived with periodic boundary conditions. In this case, we find that the fastest relaxing mode is the one where droplets alternate one by one. This relaxation mode has an eigenvalue given by the timescale obtained for a pair of droplets. All the other modes relax slower. In the limit of random position of the filled and empty droplets, the kinetic of relaxation is analytically solved by the summation of all the eigenmodes. This operation leads to an exponential decay in the early kinetic, with a time scale equal to half of the fastest mode. The same operation is performed for 
the late kinetics where the summation leads to a power law behaviour, with an exponent depending on the dimension of the system. Droplets along a line display a power-law of $1 / 2$, on a square lattice of 1 and on the nodes of a square $3 d$ lattice 3/2,as observed in our experiments (Figure 3c).
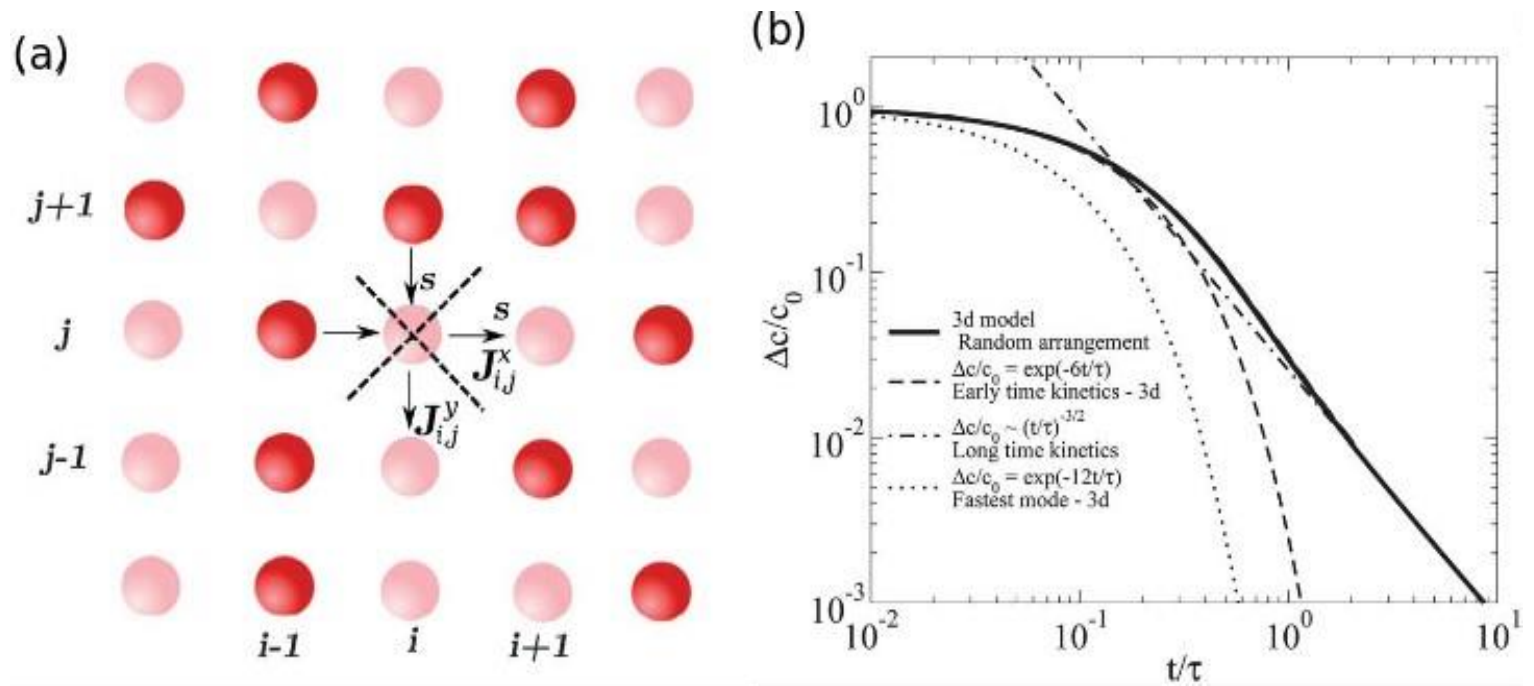

Figure 5: (a) Model for relaxation of concentration differences by permeation on a square lattice. (b) With periodic boundary conditions, the eigenmodes of the discrete diffusion equation are expressed analytically. The fastest relaxation mode (alternating droplets in both direction) decays exponentially with a time-scale linked to the exchange time scale of isolated pairs. Other modes relax slower. For a random distribution of position of filled and empty droplets, the early time relaxation is an exponential decay with a time scale twice the fastest time scale and the long time limit shows a dimension dependent power law recovered analytically. Reprinted with permission of the Royal Society of Chemistry (original publication: Y. Skhiri, P. Gruner et al. Soft Matter (2012) [11])

\section{Towards other exchange mechanisms: water transport}

In all the previous experiments, we used equal buffer concentrations to prevent the transport of water between droplets (Ostwald rippening $[7,10]$ ). But our microfluidic approach is equally usable to study this other transport problem in emulsion, namely the transport of the 
dispersed solvent between droplets. This other ageing mechanisms is a key to be controlled to guarantee the general stability of the emulsion. In addition, as it is also the basis of novel analytical platforms, where the change of relative size of droplets reveals metabolic processes down to the single cell level [10] it becomes critical to understand such processes. In a set of preliminary experiments, we consider the change of droplet sizes in droplet dispersed in fluorinated oils as a function of salt concentration differences.
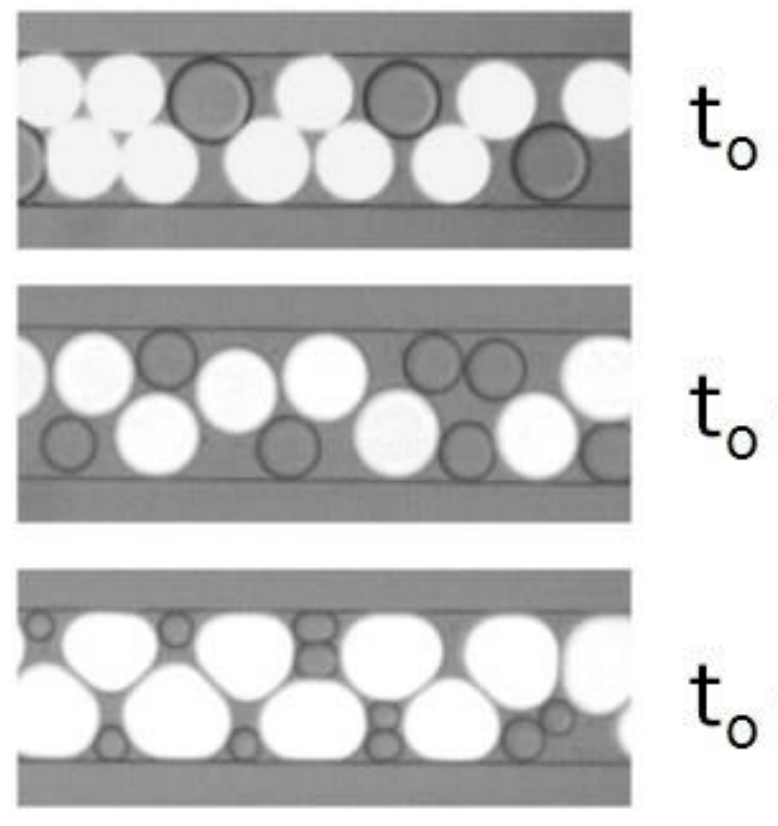

\section{$t_{0}+5 \min$}

\section{$t_{0}+20 \min$}

Figure 6: Measurement of water transport in water in oil fluorinated emulsions. Salt droplets are marked with fluorescein. The droplet volume is monitored as well as fluorescence signals. In this case, the time scale of the process in independent of surfactant concentration.

We observed that the time-scale of exchange is not affected by the surfactant concentration which indicates the the solubilization of water and organic molecules follow different mechanisms. The difference in behaviour of organic molecules and water transport relates in principle to the nature of the surfactant supramolecular assembly in the oil. The structure of the micelles solubilized in the oil will determine the affinity of the different compounds for the micelles impacting the exchange process. Experiments are currently on-going 
to determine these structure for a deeper insight into the microscopic details of the exchange processes.

\section{CONCLUSIONS}

Permeation processes in emulsions determine the long-term stability of the emulsion and are a key to be understood and controlled for applications of droplet-based microfluidics. For example, it must be ensured that all reagents and products in assays remain in the droplets for times sufficiently long compared to the time scale of the reaction considered. For storage of drugs in the form of emulsions, it must also be considered that the compounds do not diffuse across the whole emulsion. We identified here a major mechanism by which surfactants promote transport. In addition, we propose guidelines to decrease the exchange rates in emulsions: (i) optimisation of surfactant concentrations to minimize the exchange rate as the rate of exchange is linear with surfactant concentration (ii) formulation principles based on solubility alteration by additives such as BSA, as we have shown that BSA favors the partitioning of the solutes into the water phase therefore reducing exchange rates (iii) optimisation of packing and storage conditions as the exchange rates decrease with number of nearest neighbours and dimension of the system.

\section{ACKNOWLEDGMENTS}

We thank D. Pekin, L. Mazutis, V. Goust, F. Kleinschmidt, A. El Harrak, J. B. Hutchison, E. Mayot, J.-F. Bartolo, C. Rick, C. Ben Salem and Z. El Harrak and A.D. Griffiths for their contributions as well as B. Maury, S. Herminghaus, J. Bibette and N. Bremond for helpful discussions. The authors acknowledge the financial support of Region Alsace, the 'Fondation d'Entreprise EADS' and the European Commission Framework Program 6 (MiFem). Financial support from the MaxPlanck Gesellschaft and the Deutsche Forschunggemeinschaft through the Sonderforschungbereich 755 (Nanoscale Photonic Imaging) are also acknowledged.

The authors also thank the Royal Society of Chemistry for granting permission to reuse figures published in the Soft Matter Special edition 'Soft Matter Principles of Microfluidics' (Skhiri, Gruner et al. Soft Matter (2012) [11]). 


\section{REFERENCES}

1. A.B. Theberge, F. Courtois, Y. Schaerli, M. Fischlechner, C. Abell, F. Hollfelder and W.T.S. Huck. Microdroplets in microfluidics: An evolving plat-form for discoveries in chemistry and biology. Angew. Chem., Int. Ed., 49:5846-5868, (2010)

2. M.T. Guo, A. Rotem, J.A. Heyman and D.A. Weitz. Droplet microfluidics for highthroughput biological assays. Lab Chip, 12:2146-2155 (2012)

3. J.J. Agresti, E. Antipov, A.R. Abate, K. Ahn, A.C. Rowat, J.-C. Baret, M. Marquez, A. M. Klibanov, A.D. Griffiths and D.A. Weitz. Ultrahigh-throughput screening in drop-based microfluidics for directed evolution. Proc. Natl. Acad. Sci. U.S.A., 107(9):4004-4009 (2010)

4. O.J. Miller, A. El Harrak, T. Mangeat, J.-C. Baret, L. Frenz, B. El Debs, E. Mayot, M.L. Samuels, E.K. Rooney, P. Dieu, M. Galvan, D.R. Link and A.D. Griffiths. High-resolution doseresponse screening using droplet-based microfluidics. Proc. Natl. Acad. Sci. U.S.A., 109(2):378383 (2012)

5. J.-C. Baret, Y. Beck, I. Billas-Massobrio, D. Moras and A.D. Griffiths. Quantitative cell-based reporter gene assays using droplet-based microfluidics. Chem. Biol., 17(5):528-536 (2010)

6. R. Seemann, M. Brinkmann, T. Pfohl, and S. Herminghaus. Droplet-based microfluidics. Reports on Progress in Physics, 75:016601 (2012)

7. J. Bibette, F. Leal-Calderon and P. Poulin, Emulsions: basic principles, Rep. Prog. Phys., 62, 969-1033 (1999)

8. N. Bremond, A.R. Thiam and J. Bibette, Decompressing emulsion droplets favors coalescence, Phys.Rev. Lett.,100:024501 (2008)

9. J.-C. Baret, F. Kleinschmidt, A. E. Harrak and A. D. Griffiths, Kinetic Aspects of Emulsion Stabilization by Surfactants: A Microfluidic Analysis, Langmuir, 25, 6088-6093 (2009).

10. L. Boitard, D. Cottinet, C. Kleinschmitt, N. Bremond, J. Baudry, G. Yvert, and J. Bibette, Monitoring single cells bio-energetics via the coarsening of emulsion droplets, Proc. Natl. Acad. Sci. U.S.A., 109(19) 7181-7186 (2012)

11. Y. Skhiri, P. Gruner, B. Semin, Q. Brosseau, D. Pekin, L. Mazutis, V. Goust, F. Kleinschmidt, A. El Harrak, J. B. Hutchison, E. Mayot, J.-F. Bartolo, A.D. Griffiths, V. Taly and J.-C. Baret, Dynamics of molecular transport by surfactants in emulsions, Soft Matter, 8, 10618 (2012)

12. Y. N. Xia and G. M. Whitesides. Soft lithography. Annu. Rev. Mater. Sci., 28:153184 (1998)

13. S.L. Anna, N. Bontoux, H.A. Stone, Formation of dispersions using flow focusing in microchannels, Applied Physics Letters, 82(3) 364-366 (2003)

14. C. Holtze, A. C. Rowat, J. J. Agresti, J. B. Hutchison, F. E. Angile, C. H. J. Schmitz, S. Koester, H. Duan, K. J. Humphry, R. A. Scanga, J. S. Johnson, D. Pisignano and D. A. Weitz, Biocompatible surfactants for water-in-fluorocarbon emulsions, Lab Chip, 8, 1632-1639 (2008) 15. G. Woronoff, A. E. Harrak, E. Mayot, O. Schicke, O. J. Miller, P. Soumillion, A. D. Griffiths and M. Ryckelynck, New Generation of Amino Coumarin Methyl Sulfonate-Based Fluorogenic Substrates for Amidase Assays in Droplet-Based Microfluidic Applications, Anal.

Chem., 83, 2852-2857 (2011) 
16. F. Courtois, L. F. Olguin, G. Whyte, A. B. Theberge, W. T. S. Huck, F. Hollfelder and C. Abell, Controlling the Retention of Small Molecules in Emulsion Microdroplets for Use in CellBased Assays, Anal. Chem., 81, 3008-3016 (2009)

17. T. Peters, All about Albumin: Biochemistry, Genetics and Medical Applications, Academic Press, 1995.

18. B. J. Zwolinski, H. Eyring and C. E. Reese, Diffusion and membrane permability.1, J. Phys. Chem., 53, 1426-1453 (1949). 